\title{
The Induced Subgraph Order on Unlabelled Graphs
}

\author{
Craig A. Sloss * \\ Department of Combinatorics and Optimization \\ University of Waterloo, Ontario, Canada \\ csloss@math. uwaterloo.ca
}

Submitted: Jul 31, 2006; Accepted: Oct 17, 2006; Published: Oct 27, 2006

Mathematics Subject Classification: 06A07

\begin{abstract}
A differential poset is a partially ordered set with raising and lowering operators $U$ and $D$ which satisfy the commutation relation $D U-U D=r I$ for some constant $r$. This notion may be generalized to deal with the case in which there exist sequences of constants $\left\{q_{n}\right\}_{n \geq 0}$ and $\left\{r_{n}\right\}_{n \geq 0}$ such that for any poset element $x$ of rank $n$, $D U(x)=q_{n} U D(x)+r_{n} x$. Here, we introduce natural raising and lowering operators such that the set of unlabelled graphs, ordered by $G \leq H$ if and only if $G$ is isomorphic to an induced subgraph of $H$, is a generalized differential poset with $q_{n}=2$ and $r_{n}=2^{n}$. This allows one to apply a number of enumerative results regarding walk enumeration to the poset of induced subgraphs.
\end{abstract}

\section{Introduction}

The class of posets known as differential posets were first introduced and studied by Stanley [3]. Generalizations of this class of posets were studied by Stanley [4] and Fomin [1]. A number of examples of generalized differential posets are given in these papers. Another example, a poset of rooted unlabelled trees, was recently introduced by Hoffman [2]. In the present paper, we discuss a new example of a generalized differential poset, namely, the induced subgraph order on isomorphism classes of graphs. These results could potentially have applications to the analysis of dynamic algorithms in which vertices are added or removed from graphs in an unrestricted manner.

\section{Definitions and Terminology}

Let $\mathcal{P}$ be a locally finite, ranked partially ordered set with least element $\hat{0}$ and finitely many elements of each rank. For $x, y \in \mathcal{P}$, we say that $y$ covers $x$ if $x<y$ and for any

\footnotetext{
* Supported by the Natural Sciences and Engineering Research Council of Canada through its postgraduate scholarship program.
} 
$z \in \mathcal{P}$ satisfying $x \leq z \leq y$, either $x=z$ or $y=z$. We denote this by $x \lessdot y$. Let $\mathbb{K}^{\mathcal{P}}$ denote the vector space of formal, possibly infinite, linear combinations of elements of $\mathcal{P}$ over a field $\mathbb{K}$ of characteristic zero. We can define operators $U$ and $D$ on $\mathbb{K}^{\mathcal{P}}$ by

$$
U(x)=\sum_{y \in \mathcal{P}} u(x, y) y
$$

and

$$
D(x)=\sum_{y \in \mathcal{P}} d(y, x) y
$$

for some weight functions $u, d: \mathcal{P} \times \mathcal{P} \rightarrow \mathbb{K}$ such that $u(x, y), d(x, y)=0$ unless $x \lessdot y$. Let $\rho$ denote the rank function of $\mathcal{P}$. Let $\mathcal{P}_{n}$ be the set of elements of rank $n$, namely,

$$
\mathcal{P}_{n}=\{x \in \mathcal{P}: \rho(x)=n\}
$$

If $A$ is an operator on $\mathbb{K}^{\mathcal{P}}$, then its restriction to $\mathbb{K}^{\mathcal{P}_{n}}$ is denoted by $A_{n}$. (Note that for composition of operators, the notation $A B_{n}$ may be used without ambiguity, since $(A B)_{n}$ and $A\left(B_{n}\right)$ are the same operator.) Fomin [1] studied partial orders for which there exists a sequence of polynomials $\left\{f_{n}\right\}_{n \geq 0}$ for which

$$
D U_{n}=f_{n}\left(U D_{n}\right)
$$

We are typically interested in the case in which the polynomials $f_{n}$ are all linear, that is, when there exist sequences $\left\{r_{n}\right\}_{n \geq 0}$ and $\left\{q_{n}\right\}_{n \geq 0}$ such that $D U_{n}=q_{n} U D_{n}+r_{n} I$. Posets of this type are a generalization of $r$-differential posets, in which there is a constant $r$ such that $r_{n}=r$ and $q_{n}=1$ for all $n \geq 0$. Differential posets were first introduced and studied by Stanley [3]. Another special case, in which $q_{n}=1$ and $\left\{r_{n}\right\}_{n \geq 0}$ is any sequence, is the case of sequentially differential posets, also introduced and studied by Stanley [4].

A walk $C$ on a poset $\mathcal{P}$ is a sequence of poset elements

$$
C=\left(x_{1}, x_{2}, \ldots, x_{k}\right)
$$

such that either $x_{i} \lessdot x_{i+1}$ or $x_{i+1} \lessdot x_{i}$ for $1 \leq i \leq k-1$. The shape of the walk is the monomial $W=W_{k-1} W_{k-2} \ldots W_{1}$ given by

$$
W_{i}= \begin{cases}U & \text { if } x_{i} \lessdot x_{i+1} \\ D & \text { if } x_{i+1} \lessdot x_{i}\end{cases}
$$

The displacement of a walk of shape $W$ is the number of instances of $U$ in $W$ minus the number of instances of $D$ in $W$. The length of a walk is the total number of $U$ 's and $D$ 's in $W$. The weight of the walk $C$ is the product

$$
w(C)=\prod_{1 \leq i \leq k-1} w\left(x_{i}, x_{i+1}\right)
$$


where

$$
w\left(x_{i}, x_{i+1}\right)= \begin{cases}u\left(x_{i}, x_{i+1}\right) & \text { if } x_{i} \lessdot x_{i+1} \\ d\left(x_{i+1}, x_{i}\right) & \text { if } x_{i+1} \lessdot x_{i}\end{cases}
$$

Let $\mathcal{C}(x \stackrel{W}{\rightarrow} y)$ denote the set of all walks of shape $W$ which start at $x$ and end at $y$. We are interested in studying the sum of weights over walks from $x$ to $y$ of a given shape $W$, namely,

$$
e(x \stackrel{W}{\rightarrow} y):=\sum_{C \in \mathcal{C}(x \stackrel{W}{\rightarrow} y)} w(C) .
$$

If $W=U^{n}$, this notation is shortened to $e\left(x \stackrel{U^{n}}{\rightarrow} y\right)=e(x \rightarrow y)$. A further simplification of notation is $e(\hat{0} \rightarrow x)=e(x)$. The key observation connecting these numbers to the algebraic structure of a generalized differential poset is that

$$
W x=\sum_{y \in \mathcal{P}} e(x \stackrel{W}{\rightarrow} y) y,
$$

so answering the algebraic question of how to compute $W x$ will answer combinatorial questions about enumeration of walks. It is often helpful to introduce the bilinear form $\langle\cdot, \cdot\rangle$ given by

$$
\langle x, y\rangle=\delta_{x, y}= \begin{cases}1 & \text { if } x=y \\ 0 & \text { if } x \neq y\end{cases}
$$

extending linearly in both arguments to vectors of finite support. With this notation, we have

$$
e(x \stackrel{W}{\rightarrow} y)=\langle y, W x\rangle
$$

\section{The Induced Subgraph Order on Unlabelled Graphs}

Let $\mathcal{V}$ denote the set of isomorphism classes of simple graphs. Define a partial order on $\mathcal{V}$ by $G \leq H$ if and only if $G$ is isomorphic to an induced subgraph of $H$. In this order, the covering relation is given by $G \lessdot H$ if and only if there exists a vertex of $H$ which, when deleted, leaves $G$. Equivalently, $G \lessdot H$ if and only if there exists a subset $S$ of vertices of $G$ such that when a new vertex $v$ is added to $G$ such that the neighbourhood of $v$ is $S$, the result is $H$. (We denote the graph obtained by adding the vertex $v$ to $G$ such that its neighbourhood is $S$ by $G \boxplus S$.) From this, by taking $\rho(G)$ to be the number of vertices of $G$, we see that $\mathcal{V}$ is ranked, and its least element is the graph with no vertices.

If $G \lessdot H$, we can define weights on the cover relations as follows. Let $d(G, H)$ denote the number of vertices $v$ of $H$ for which $H \backslash\{v\}=G$. Let $u(G, H)$ denote the number of subsets $S$ of vertices of $G$ such that $G \boxplus S=H$. These are natural cover weights to use in the sense that the weight of a walk on this poset will correspond to the number of ways one graph may be transformed into another through a sequence of additions and deletions of vertices. Moreover, with these weights, for $\rho(G)=n$ and a fixed labelling 
$\{1, \ldots, n\}$ of the vertices of $G$, we can write $U$ and $D$ as

$$
U(G)=\sum_{H \in \mathcal{V}} u(G, H) H=\sum_{S \subseteq\{1, \ldots, n\}} G \boxplus S
$$

and

$$
D(G)=\sum_{H \in \mathcal{V}} d(H, G) H=\sum_{i \in\{1, \ldots, n\}} G \backslash\{i\} .
$$

Our main theorem concerning the poset $\mathcal{V}$ is as follows.

Theorem $3.1 \mathcal{V}$, with cover weights $u$ and $d$ defined as above, is a generalized differential poset with

$$
D U_{n}=2 U D_{n}+2^{n} I_{n}
$$

Proof: Let $G \in \mathcal{V}$ be such that $\rho(G)=n$. Fix a labelling $\{1, \ldots, n\}$ of the vertices of $G$. Then

$$
\begin{aligned}
D U(G) & =D\left(\sum_{S \subseteq\{1, \ldots, n\}} G \boxplus S\right) \\
& =\sum_{S \subseteq\{1, \ldots, n\}} \sum_{i \in\{1, \ldots, n, v\}}(G \boxplus S) \backslash\{i\} \\
& =\sum_{S \subseteq\{1, \ldots, n\}} \sum_{i \in\{1, \ldots, n\}}(G \boxplus S) \backslash\{i\}+2^{n} G,
\end{aligned}
$$

and

$$
\begin{aligned}
U D(G) & =U\left(\sum_{i \in\{1, \ldots, n\}} G \backslash\{i\}\right) \\
& =\sum_{i \in\{1, \ldots, n\}} \sum_{S \subseteq\{1, \ldots, n\}, i \notin S}(G \backslash\{i\}) \boxplus S .
\end{aligned}
$$

Note that if $i \notin S$, then the graphs $(G \backslash\{i\}) \boxplus S$ and $(G \boxplus S) \backslash\{i\}$ are the same, so

$$
U D(G)=\sum_{i \in\{1, \ldots, n\}} \sum_{S \subseteq\{1, \ldots, n\}, i \notin S}(G \boxplus S) \backslash\{i\} .
$$

Furthermore, if $i \notin S$, then the graph $(G \boxplus S) \backslash\{i\}$ is the same as the graph $(G \boxplus(S \cup$ $\{i\}) \backslash\{i\}$, so

$$
\sum_{i \in\{1, \ldots, n\}} \sum_{S \subseteq\{1, \ldots, n\}, i \notin S}(G \boxplus S) \backslash\{i\}=\sum_{i \in\{1, \ldots, n\}} \sum_{S \subseteq\{1, \ldots, n\}, i \in S}(G \boxplus S) \backslash\{i\} .
$$

From this, we obtain

$$
U D(G)=\frac{1}{2} \sum_{i \in\{1, \ldots, n\}} \sum_{S \subseteq\{1, \ldots, n\}}(G \boxplus S) \backslash\{i\}=\frac{1}{2} \sum_{S \subseteq\{1, \ldots, n\}} \sum_{i \in\{1, \ldots, n\}}(G \boxplus S) \backslash\{i\},
$$


hence $D U(G)=2 U D(G)+2^{n} G$ for all $G$ such that $\rho(G)=n$, so $D U_{n}=2 U D_{n}+2^{n} I_{n}$.

It is of interest to note that the induced subgraph order is an example of a generalized differential poset in which the multiplicative parameter $q_{n}$ is nontrivial - though the theory developed by Fomin allows $\left\{q_{n}\right\}_{n \geq 0}$ to be an arbitrary sequence, the known examples of generalized differential posets all have $q_{n}=1$.

\section{Enumerative Results}

Having proven that the induced subgraph order is a generalized differential poset, we now have at our disposal all the algebraic and enumerative results pertaining to generalized differential posets. This section provides a sample of those results. By Theorem 3.1, the operators $U$ and $D$ on $\mathcal{V}$ satisfy relations of the form of Equation (1), where the sequence $\left\{f_{n}\right\}_{n \geq 0}$ is given by

$$
f_{n}(t)=2 t+2^{n}
$$

Most of the results in this section are stated in terms of repeated composition of polynomials from the sequence $\left\{f_{n}\right\}_{n \geq 0}$, so we introduce the notation

$$
f_{a \leftarrow b}:=f_{a} \circ f_{a-1} \circ \cdots \circ f_{b}
$$

for $a \geq b$, with the convention that $f_{a \leftarrow b}=0$ if $a<b$. Observe that for the sequence $\left\{f_{n}\right\}_{n \geq 0}$ corresponding to $\mathcal{V}$, we have

$$
f_{a \leftarrow b}(t)=2^{a-b+1} t+(a-b+1) 2^{a} .
$$

Our first tool is the following result of Fomin, which appears as part of Lemma 1.4.8 in $[1]$.

Lemma 4.1 (Fomin) Let $k \geq 1$. Then

$$
D U_{n}^{k}=U^{k-1} f_{n+k-1 \leftarrow n}\left(U D_{n}\right)
$$

and

$$
D^{k} U_{n}=f_{n \leftarrow n-k+1}\left(U D_{n-k+1}\right) D_{n}^{k-1} .
$$

We can use this lemma to write a special class of monomials $W$ in a convenient canonical form. If

$$
W=U^{a_{m}} D U^{a_{m-1}} D \ldots D U^{a_{1}} D U^{a_{0}}
$$

for integers $a_{i} \geq 0$, we say $W$ is an above-word if $\sum_{0 \leq i \leq k} a_{i}>k$ for all $0 \leq k \leq m$. Monomials of this type correspond to walks which, in rank, do not go below their starting points. With this definition in hand, we can prove the following.

Theorem 4.2 Let $\mathcal{P}$ be a generalized differential poset, and let

$$
W=U^{a_{m}} D U^{a_{m-1}} D \ldots D U^{a_{1}} D U^{a_{0}}
$$


be an above-word. Let $b_{i}=\sum_{0 \leq j \leq i} a_{j}-i$. Then there exists a polynomial $g_{W, n}$ such that $W_{n}=U^{b_{m}} g_{W, n}\left(U D_{n}\right)$, namely

$$
g_{W, n}=\prod_{0 \leq i \leq m-1} f_{n+b_{i}-1 \leftarrow n} .
$$

Proof: Use induction on $m$. The base case, $m=0$, is trivial. Suppose $m>0$ and that the result holds for all smaller values of $m$. By Lemma 4.1,

$$
W_{n}=U^{a_{m}} D U^{a_{m-1}} D \ldots D U^{a_{1}} U^{a_{0}-1} f_{n+a_{0}-1 \leftarrow n}\left(U D_{n}\right)=W^{\prime} f_{n+a_{0}-1 \leftarrow n}\left(U D_{n}\right)
$$

where $W^{\prime}=U^{a_{m}} D U^{a_{m-1}} D \ldots D U^{a_{1}+a_{0}-1}$. Let $a_{0}^{\prime}=a_{1}+a_{0}-1$, and $a_{i}^{\prime}=a_{i+1}$ for $m-1 \leq i \leq 1$. Let $b_{i}^{\prime}=\sum_{0 \leq j \leq i} a_{i}^{\prime}-i$. Note that

$$
\begin{aligned}
b_{i}^{\prime} & =a_{0}+a_{1}-1+\sum_{1 \leq j \leq i} a_{j+1}-i \\
& =\sum_{0 \leq j \leq i+1} a_{j}-i+1 \\
& =b_{i+1},
\end{aligned}
$$

so, since $W$ is an above-word, $b_{i}^{\prime}>0$ for $0 \leq i \leq m-1$. Hence $W^{\prime}$ is also an above-word, so applying the inductive hypothesis,

$$
\begin{aligned}
W_{n} & =U^{b_{m-1}^{\prime}} \prod_{0 \leq i \leq m-2} f_{n+b_{i}^{\prime}-1 \leftarrow n}\left(U D_{n}\right) f_{n+a_{0}-1 \leftarrow n}\left(U D_{n}\right) \\
& =U^{b_{m}} \prod_{0 \leq i \leq m-2} f_{n+b_{i+1}-1 \leftarrow n}\left(U D_{n}\right) f_{n+a_{0}-1 \leftarrow n}\left(U D_{n}\right) \\
& =U^{b_{m}} \prod_{0 \leq i \leq m-1} f_{n+b_{i}-1 \leftarrow n}\left(U D_{n}\right) .
\end{aligned}
$$

Thus, by induction, the polynomial $g_{W, n}$ exists and is equal to the given formula.

Theorem 4.2 provides a method of computing $e(\hat{0} \stackrel{W}{\rightarrow} x)$ for any above-word $W$ and $x \in \mathcal{P}$ whose rank is equal to the displacement of $W$. Namely,

$$
\begin{aligned}
e(\hat{0} \stackrel{W}{\rightarrow} x) & =\langle x, W \hat{0}\rangle \\
& =\left\langle x, U^{\rho(x)} g_{W, 0}\left(U D_{0}\right) \hat{0}\right\rangle \\
& =\left\langle x, g_{W, 0}(0) U^{\rho(x)} \hat{0}\right\rangle,
\end{aligned}
$$

since $D \hat{0}=0$. Thus, we obtain the following.

\section{Corollary 4.3}

$$
e(\hat{0} \stackrel{W}{\rightarrow} x)=e(x) \prod_{0 \leq i \leq m-1} f_{b_{i}-1 \leftarrow 0}(0) .
$$


In particular, for the poset of induced subgraphs, we obtain this formula.

\section{Corollary 4.4}

$$
e(\hat{0} \stackrel{W}{\rightarrow} x)=e(x) 2^{\sum_{0 \leq i \leq m-1}\left(b_{i}-1\right)} \prod_{0 \leq i \leq m-1} b_{i}
$$

Note that for fixed $W$, these values depend only on $e(x)$, which, for $\rho(x)=n$, is the number of graphs on vertices $\{1, \ldots, n\}$ which are in the isomorphism class $x$.

To compute $e(y \stackrel{W}{\rightarrow} x)$ for $y, x \in \mathcal{P}$ and above-word $W$ of displacement $\rho(x)-\rho(y)$, we can make use of the diagonalization of the operator $U D_{n}$ carried out by Fomin [1]. Namely, if $\rho(y)=n$, find a basis for $\mathbb{K}^{\mathcal{P}_{n}}$ consisting of eigenvectors of $U D_{n}$. Writing $y$ in terms of this basis, one can easily compute $g_{W, n}\left(U D_{n}\right) y$ by evaluating the polynomial $g_{W, n}$ at the eigenvalues of $U D_{n}$.

\section{Extensions}

Matthew Walsh, in a private communication, observed that replacing the ground set of $\mathcal{V}$ with the set of unlabelled multigraphs in which each edge appears with multiplicity at most $k-1$ gives a generalized differential poset with $D U_{n}=k U D_{n}+k^{n} I_{n}$. (The proof proceeds as the proof of Theorem 3.1, with the natural modifications.) Thus, we have examples of generalized differential posets for which the sequence $\left\{q_{n}\right\}_{n \geq 0}$ is a constant $k$, for any positive integer $k$.

\section{References}

[1] S. Fomin, Duality of graded graphs, J. Algebraic Combin. 3 (1994), 357-404.

[2] M. E. Hoffman, Combinatorics of rooted trees and Hopf algebras, Trans. Amer. Math. Soc. 355 (2003), 3795-3811.

[3] R. P. Stanley, Differential posets, J. Amer. Math. Soc. 1 (1988), 919-961.

[4] R. P. Stanley, Variations on differential posets, Invariant Theory and Tableaux (D. Stanton, ed.), Springer-Verlag, 1990, pp. 145-165. 